\title{
THE MAMMAE AS ENDOCRINE ORGANS
}

THAT the mammary glands have a dual function is not yet altogether accepted by physiologists or clinicians in general. Evidence, both experimental and clinical, is gradually accumulating to indicate that the mammae may have an endocrine function; but this evidence is not as concise and unimpeachable as might be desired. The whole matter still must be considered as sub judice.

With the growing appreciation of the internal secretory value of many parts of the body, not a few of which are well known to have a dual secretory function-both internal and external, and the intimacy of the mammary glands with other organs of internal secretion (especially the ovaries, pituitary and possibly the thyroid) it may not be amiss to recall a number of the indications that lead some to believe with us that the mammary glands are indeed a part of the endocrine system, and that this phase of their functional activities is deserving of study and the resulting knowledge of much wider clinical application.

First it is clear to all that the functional activity of the mammary glands is influenced by numerous hormonic means, for their occasional transient functional activity at birth (in the production of the socalled "witches' milk"), their growth at puberty, their frequent "sympathetic association" with the menses, their relation to the sex manifestations and last, and most important of all, their response to chemical stimuli obviously of intrauterine origin, surely constitutes a series of phenomena very similar to, if indeed not actually due to, internal secretory reactions.

Not so long ago Schil selected the manifestations 
of mammary evolution and physiology as the basis of a masterly study (These de Nancy, 1912) and therein sets forth his conclusions that mammary evolution is properly divided into six stages: (1) The stage of development, divided into an organogenetic phase and a phase in which there is secretory activity (the lactation in the new-born already mentioned). This is believed to be due to a hormone passing from the mother which also probably is concerned in activating mammary secretion in the mother. (2) The prepubertial stage of functional inactivity. (3) The stage of puberty, characterized by a considerable increase in glandular parenchyma which is dependent upon the maturation of the corpora lutea and obviously the result of internal secretory activities.* The gravid stage, divided into the early period during which the glands attain their full development and a "phase glandulaire gravidique" which follows complete development and is characterized by secretory activity (colostrum). It is suggested that the first. part of this stage is controlled by the corpus luteum of pregnancy through a hormone produced in it, while the latter part results from a chemical messenger from the fetus itself, the placenta or possibly a myometrial gland. (5) The postpartum stage includes the period of lactation initiated during pregnancy by an endogenous stimulus and maintained by mechanical means-nursing. The phase of post-

*It has been repeatedly demonstrated that the ovaries influence mammary development. For instance Bouin and Ancel (Jour. de physiol. et de pathol. general; 1911, xiil, 31) showed that ovariotomy resulted in a practical disappearance of the mammae, while on the successful grafting of a piece of ovary, the lost glands reappeared. Again O'Donoghue (Jour. Physiol. 1911, xliii, p. xvi) and others have ruptured the ripe Graafian follicles artificially, thereby making a noticeable increase both in mammary growth and activity. 
partum retrogression is merely due to an absence of stimuli. Finally there is (6) the senile stage, associated with involution, and corresponding to the period when the gonads atrophy and their stimuli no longer are active.

All these comparatively well known facts serve to emphasize the interrelation of the mammary glands with endocrine function and while this evidence is none too positive, for it does not prove these glands to have an internal secretion, at least it classes them intimately with other organs known to have distinct endocrine function.

Occasionally our knowledge of the physiological activity of a given endocrine organ has resulted from its experimental removal or, more often, from the administration of extracts of it. The internal secretory character of the pituitary was demonstrated in this manner by Schaefer and his associates in 1895. Practically all the clinical value of the adrenal medullary principle resulted from gland feeding, though, of course, much physiological work has been done in other ways. Be this as it may, mammary gland feeding is not without its influence upon the organism, and in addition to a homostimulant action which is to be expected on general principles, it is of interest to recall that the chief organs affected by prolonged mammary therapy are the ovaries.

Numerous reports indicate that mammary extract exerts an antagonist effect upon ovarian endocrine activity, and its use in hyperovarism, menorrhagia and even fibroids seems to have been accompanied by indubitable results. The writings of Bell, Shober, Feodoroff, Battuaud, Luncz, Carnot and a number of 
others* seem to contain within them sufficient clinical proof that this remedy is not without clinical value. We have had occasion to prescribe it in, perhaps, forty cases and the results have confirmed our belief that there is a principle in mammary substance which antagonizes ovarian activity, reduces pelvic congestion and stops local bleeding. One case recently demonstrated this unusually well: A well advanced and inoperable carcinoma uteri, which was the source of constant hemorrhage, was treated for a short time with 30 grains of mammary extract daily. The hemorrhage was reduced to a minimum, there was a corresponding increase in strength and the patient was even led to hope that she would be cured. This influence was merely temporary, the hemorrhage returning shortly after the mammary gland was stopped; but was this proof enough that the antihemorrhagic effect was due to the remedy and not to suggestion, other treatment or coincidence?

Looking at this from another angle the evidence is not so complete. The breasts are rarely removed for therapeutic purposes in women early in ovarian activity; though there seems to be some proof that this operation is not without influence upon endocrine function. We know, however, that the function of ovulation may be retarded and sometimes entirely stopped by prolonged lactation, and the writer has seen a case of premature menopause in a young woman which resulted in our opinion solely from an inordinately prolonged period of lactation-four years!

Not uncommonly we find women with large mammary development whose menses are noticeably

*Including also the article by Briggs in this issue, page 188. 
scanty, though this is far from a rule. On the other hand in spayed dairy cows it is well established that the period of lactation is prolonged. It is also well known that pregnant cows as a rule show a tendency to lessen their milk producing powers soon after pregnancy takes place.

There is opportunity for some animal experimentation to demonstrate the accuracy or futility of our views that the mammae have internal secretory powers; and it should not present great difficulty to remove the mammae in a number of animals and watch the functional and histologic changes in other endocrine organs, notably the ovaries. From a clinical standpoint, too, there should be numerous experiences to substantiate this position, or not, as the case may be; and it is hoped that future issues of this journal may contain scientific evidence on this subject, since it is intimately concerned with certain phases of clinical gynecology and if the endocrine function of the mammae is proved we shall have an explanation of the clinical experiences with mammary therapy already referred to.

HENRY R. HARROWER. 